\section{ONOMÁVAEIN}

Revista semestral de lingüística, filología y traducción
PONTIFICIA UNIVERSIDAD

\title{
Traducción de calidad de webs hoteleras: discurso interpersonal e implicación del cliente*
}

Quality translation of hotel websites:

interpersonal discourse and customer's engagement

\section{Francisca Suau Jiménez \\ Universitat de València \\ España}

\section{(c) $\odot$}

Francisca Suau Jiménez: Instituto Interuniversitario de Lenguas Modernas Aplicadas (IULMA), Universitat de València, España. | Correo electrónico: francisca.suau@uv.es 


\section{Resumen}

Este trabajo sugiere la necesaria traducción de calidad o traducción "pragmática" de páginas web de hotel (Durán Muñoz, 2011), según el principio de localización (Pym, 2004; Dunne, 2006) y según el discurso interpersonal, siguiendo la clasificación de Hyland y Tse (2004), con objeto de preservar la persuasión del lector/cliente. Para ello se ha analizado un subcorpus de páginas web de hotel que ofrecen traducción (COMETVAL, Corpus Multilingüe en Turismo, Universitat de València), traducidas de inglés a español, de hoteles de EEUU y Reino Unido, teniendo en cuenta el patrón interpersonal del español en géneros turísticos (Mapelli, 2008; Suau-Jiménez, 2012a, 2012b). El estudio se contrasta con un análisis discursivo similar de fichas de hotel en español, extraídas de los portales Booking y TripAdvisor. Los resultados sugieren que una traducción de calidad de sitios web de hotel no solo debe incluir las funciones retóricas pertinentes sino también el discurso interpersonal. De no ser así, la persuasión y la implicación del lector/cliente puedan resultar dañadas, como ocurre en las fichas de los mencionados buscadores analizados, pudiendo quedar reducida la especificidad del hotel al tiempo que no se satisfacen las crecientes expectativas de implicación de los viajeros en este género digital. Las implicaciones para la industria turística apuntan hacia la necesidad de mayor inversión en diseño y traducciones de calidad de las webs como refuerzo de la promoción hotelera. Asimismo, este estudio abre nuevas vías de investigación en la traducción de otros géneros de promoción.

Palabras clave: webs de hotel; discurso interpersonal; traducción de calidad; persuasión; localización.

\section{Abstract}

This research points towards the need of a quality or "pragmatic" translation of hotel websites (Durán Muñoz, 2011), following the principle of localization (Pym, 2004; Dunne, 2006) and taking into account the interpersonal (interactional) discourse classification by Hyland and Tse (2004), in order to preserve the reader's/ customer's persuasion. A sub-corpus of translated US and UK hotel websites from English into Spanish, obtained from COMETVAL (Corpus Multilingüe en Turismo, Universitat de València), has been analyzed following the Spanish interpersonal pattern for tourism genres (Mapelli, 2008; Suau-Jiménez, 2012a, 2012b). This study has been contrasted with a similar discursive analysis of hotel records in Spanish extracted from the Booking and TripAdvisor portals. Results suggest that a quality translation should not only include the necessary rhetorical functions but also the interpersonal discourse. Otherwise, persuasion and reader's/customer's engagement can be damaged, as happens with hotel records in the two analyzed portals. Also, the hotel's specificity can be reduced as well as travellers' increasing demand for engagement in hotel websites. Implications for the tourism industry suggest that hotels should increase investments in the design and quality translation of their websites in order to reinforce hotels' promotion. Besides, this study opens up a new research perspective related to the translation of promotion websites.

Keywords: hotel website; interpersonal discourse; persuasion; quality translation; localization.

* Este trabajo es resultado del Proyecto I+D: Análisis léxico y discursivo de corpus paralelos y comparables (español-inglés-francés) de páginas electrónicas de promoción turística; Ref. FFI2011 (2011-2014). Ministerio de Economía y Competitividad, España. 


\section{La traducción de calidad o traducción pragmática de páginas web de hotel}

Las páginas o sitios web de hotel constituyen un elemento clave en la promoción turística. El auge imparable del turismo 2.0, la industria de los viajes y su internacionalización las ha convertido en cruciales, ya que es el propio viajero quien escoge sus destinos y alojamientos y las usa como plataforma de información y comunicación con el hotel. Desde que existe internet, ha decrecido la figura del agente de viajes o intermediario, que era anteriormente el referente principal en asesoramiento al viajero. Ahora es éste quien rastrea internet en busca de la mejor opción, con lo cual han cobrado importancia cibergéneros turísticos tales como guías, portales y foros de viajeros, que ofrecen opiniones directas, así como sitios web de alojamientos y restaurantes. Estos géneros, entre ellos la web de hotel, están diseñados de forma multimodal (incluyen texto, imágenes y vídeos) para lograr una información completa y están concebidos desde la persuasión, de acuerdo con las necesidades individuales sociocognitivas cada vez más específicas y exigentes de unos usuarios bien formados e informados. Estas necesidades o expectativas llegan a través de diversos sistemas de "user-generated-content-UGC" (Cheng y Ab Hamid, 2011), o contenidos generados por los usuarios, como comentarios o reseñas de viajeros en la propia web o en foros y redes sociales, y conforman así un sistema comunicativo simbiótico. Las webs hoteleras, siguiendo el principio de la usabilidad, necesitan así rediseñarse de forma constante para ajustarse a las expectativas de los clientes en una combinación triangular que incluye tecnología, proceso y factor humano (Chen y Popovich, 2003).

Este factor humano estaría ligado a la comunicación y a cómo se desarrolla ésta. Según Pierini (2007: 2), como resultado de la internacionalización del turismo y de la consiguiente localización de las webs, esta comunicación se da simultáneamente en varias lenguas, por lo cual la traducción de las webs es una práctica necesaria. La localización como actividad que incluye necesariamente aspectos discursivos se ha tratado en diversos trabajos (Jiménez Crespo, 2008, 2011, 2013). Esta traducción se entiende como una actividad que incluye diversos campos: Ia comunicación intercultural, la publicidad, el márketing, las TIC y la propia teoría de la traducción, incluyendo la lingüística aplicada y el análisis del discurso aplicado a la práctica traductológica (Suau-Jiménez, 2014b). Como hemos dicho, este proceso está ligado a la localización, entendida, según las propuestas de Esselynk (2000) y Pym (2004) que recoge Pierini (2007), como el proceso de traducción del contenido lingüístico, que incluye un $75 \%$ del léxico específico junto con la adaptación del contenido y del estilo para ser mejor recibidos por los lectores de la lengua de llegada (además de otros procesos de adaptación cultural del producto al mercado local, como son los pesos, medidas, monedas, documentos de apoyo, imágenes, etc.). La falta de traducción o la traducción deficiente de las webs comerciales incide negativamente en los buenos resultados económicos de las empresas e industrias que utilizan webs para promocionar sus productos y servicios, a veces hasta con un 20\% en pérdidas de ventas (Medina Reguero y Ramírez, 2014).

La localización de páginas web, como parte de la internacionalización del turismo, está, por lo tanto, fuertemente ligada a la traducción, y ésta, a la actividad intercultural propia de este sector. Según Dunne (2006) y Pym (2012), la localización de webs incluye la traducción del contenido textual a la lengua y convenciones de la audiencia local o "target locale". Así pues, responde sobre todo a la adaptación de los productos a la lengua y cultura de Ilegada. Como indica Durán Muñoz (2012: 103), interculturalidad e internacionalización hacen de la traducción una herramienta de mediación imprescindible para resolver los posibles problemas de comunicación entre culturas locales y extranjeras. Sin ella no 
siempre sería posible esta comunicación, y, añadimos, tampoco lo sería quizá el fin promocional y persuasivo de ciertos géneros turísticos. Por lo tanto, la calidad en la traducción de las guías turísticas, las webs de establecimientos hoteleros, de restauración y de otros servicios paralelos debería ser un principio ineludible para la industria turística. Sin embargo, esto no siempre es así por múltiples razones, como el bajo presupuesto que las empresas dedican a la traducción de calidad de una web o los problemas técnicos implícitos (Bass, 2006). Todo ello nos lleva a reflexionar brevemente sobre qué significa una traducción de calidad.

En traducción, la calidad va a menudo pareja a la adecuación (Durán Muñoz, 2012: 104), en el sentido que le aplica Nord (1997) de ser apropiada al fin comunicativo definido en el propio encargo de dicha traducción. En otras palabras, la calidad es un concepto variable pero siempre sometido al fin que se persigue con la traducción de un texto. Según sugiere Nobs (2003), la traducción turística de calidad ha de cumplir con dos objetivos: ser funcional y pragmática, es decir, ajustarse al encargo requerido por el cliente pero también adecuarse a la lengua meta en sus aspectos estilísticos y culturales para que tenga una buena recepción por los lectores.

En cualquier caso, creemos que esta adecuación ha de ser ante todo pragmática, en el sentido que apunta Durán Muñoz (2011: 379-381):

En cuanto a la adecuación pragmática, la información que se ofrece deberá ser útil y accesible para un receptor del texto meta, deberá ajustarse a las normas y convenciones de la cultura meta, deberá ofrecer un estilo y un registro normal en la cultura de llegada para ese tipo textual y, por último, deberá contar con la terminología y fraseología propias del campo de especialidad en cuestión.

Dentro de esta adecuación pragmática tiene especial importancia el discurso interpersonal entendido como el conjunto de elementos lingüísticos, tanto de contenido como metadiscur- sivos, que responden a la función interpersonal (Halliday, 1986) y que establecen por tanto un diálogo, explícito o implícito, entre el autor de un texto y el lector, siguiendo un propósito preestablecido. El discurso interpersonal, tal como aquí lo definimos, supone una evolución del concepto original de metadiscurso (Vande Kopple, 1985; Crismore y otros, 1993; Hyland, 2005; Hyland y Tse, 2004; Hyland, 2008), que empezó aplicándose solamente al discurso académico a través de un patrón de marcadores propios del inglés (Hyland y Tse, 2004). Tras diversos estudios y su aplicación a otras disciplinas no académicas, se han eliminado unos marcadores e incorporado otros, tanto de contenido como metadiscursivos, modificando el patrón interactivo que proponían Hyland y Tse (2004) y concluyendo que el género, la disciplina y la lengua son variables que conforman este patrón, que es flexible (Suau-Jiménez, 2011, 2012a, 2012b). Así lo demuestran varios estudios en el campo del turismo (Pierini, 2007; Mapelli, 2008; Suau-Jiménez y Dolón-Herrero, 2007; Suau-Jiménez, 2012a, 2012b, 2014a; Edo Marzá, 2012).

\section{Hipótesis y objetivo}

La hipótesis y pregunta de investigación de la que partimos es si los hoteles deberían ofrecer una traducción de calidad o traducción pragmática de su página o sitio web, teniendo en cuenta el discurso interpersonal, para preservar así la persuasión, en lugar de dirigir al lector/cliente hacia traductores automáticos o hacia portales tipo Booking o TripAdvisor cuando esta traducción no existe. Los traductores automáticos ofrecen traducciones deficientes, y los portales de servicios turísticos solo proporcionan una ficha tipo, donde únicamente se incluyen funciones retóricas básicas así como algunos datos objetivos. Esta carencia de traducciones propias en las webs podría llevar implícito un peor resultado de la promoción y del márketing de los hoteles, ya que las fichas de portales turísticos no incluyen aspectos discur- 
sivos interpersonales sobre los que recae una parte importante de la función persuasiva, de la implicación del cliente, de la especificidad del hotel y de la localización.

El objetivo ha sido, pues, analizar, desde el punto de vista de la localización y especialmente del discurso interpersonal y su aplicación a la traducción, los textos que aparecen en varios sitios web, sacados de 200 hoteles del Reino Unido y de EEUU, compilados en el corpus COMETVAL (Corpus Multilingüe en Turismo, Universitat de València). Nos hemos centrado en los que ofrecen traducción al español y los hemos comparado con varias fichas descriptivas de hoteles en español, en portales como Booking o TripAdvisor, para extraer así conclusiones pertinentes. La hipótesis concreta que hemos querido comprobar es la de que parece que estas fichas no tienen en cuenta estrategias discursivas interpersonales esenciales para la persuasión y la localización, las cuales difieren de una lengua a la otra por tener un sustrato sociocultural y lingüístico propio (Suau-Jiménez, 2012a, 2012b) y, por lo tanto, no pueden sustituir adecuadamente a las webs de hotel para persuadir al cliente.

\section{Metodología}

La metodología que se ha aplicado a la investigación ha sido el modelo o clasificación del metadiscurso interpersonal (interactivo) de Hyland y Tse (2004). Se ha seguido el patrón de marcadores agrupados según la voz del autor como autoridad (stance) y la voz del lector como implicación (engagement), basado en el trabajo de White (2003) sobre las voces en el discurso no literario y desarrollado por Hyland (2008) para el discurso y los géneros académicos, que reproducimos a continuación:

\begin{tabular}{|c|c|c|}
\hline \multirow{6}{*}{$\begin{array}{l}\text { PATRÓN } \\
\text { INTERPERSONAL O } \\
\text { INTERACTIVO } \\
\text { (Hyland y Tse, 2004; } \\
\text { Hyland, 2008) }\end{array}$} & $\begin{array}{l}\text { STANCE } \\
\text { (VOZ DEL AUTOR) }\end{array}$ & $\begin{array}{l}\text { ENGAGEMENT } \\
\text { (VOZ DEL LECTOR) }\end{array}$ \\
\hline & Hedges (atenuadores) & $\begin{array}{l}\text { Reader pronouns } \\
\text { (pronombres del lector) }\end{array}$ \\
\hline & Boosters (redizadures) & Directives (directivos) \\
\hline & Attitude markers & Ousctionc (nroguntac alloctor) \\
\hline & Self-Mention (automención) & $\begin{array}{l}\text { Knowledge references } \\
\text { (referencias al conocimiento) }\end{array}$ \\
\hline & & Asides (apartes) \\
\hline
\end{tabular}

Se ha adaptado a este modelo el patrón interpersonal del español para géneros turísticos de internet y sus marcadores, surgido de varios trabajos (Pierini, 2009; Mapelli, 2008) y desarrollado por Suau-Jiménez y Dolón-Herrero (2007) y Suau-Jiménez (2012a y 2012b) para los géneros de promoción en esta disciplina. Estas últimas autoras han analizado un corpus extenso de géneros turísticos en español e inglés, y se ha podido comprobar que existe variación en el tipo y la cantidad de marcadores interpersonales, tanto para la voz del autor como del lector. Esta variación ha dado lugar a un patrón interpersonal distinto, con propiedades caracterizadoras y que depende del género, de la disciplina y de la lengua, incluyendo a la vez marcadores de contenido y marcadores metadiscursivos. Todo ello ha llevado a concluir que el discurso interpersonal es un concepto y un modelo de análisis útil pero variable que se ha de estudiar en cada caso concreto, y que ha evolucionado notablemente desde sus inicios. En este trabajo aplicamos el patrón metadiscursivo propio de páginas de hotel para el español (Suau-Jiménez y Dolón-Herrero, 2007; Suau-Jiménez, 2012a y 2012b). Este patrón se caracteriza por la existencia, basada en el análisis del género Página Web 
Institucional de Promoción Turística, dentro del corpus COMETVAL, de los siguientes marcadores y categorías interpersonales, ordenados según su frecuencia, de mayor a menor:

\begin{tabular}{|c|c|c|}
\hline & VOZ DEL AUTOR & VOZ DEL LECTOR \\
\hline \multirow{4}{*}{$\begin{array}{l}\text { PATRÓN } \\
\text { INTERPERSONAL PARA } \\
\text { GÉNEROS TURÍSTICOS } \\
\text { DE PROMOCIÓN EN } \\
\text { INTERNET } \\
\text { Suau-Jiménez (2012a) }\end{array}$} & Realzadores (78'46\%) & Directivos (3'04\%) \\
\hline & Atenuadores (7’ı\%) & Pronombres del lector (2'57\%) \\
\hline & Auto-mención (6’92\%) & \\
\hline & Marc. de Actitud (0’57\%) & \\
\hline
\end{tabular}

Este modelo muestra una clara preponderancia de los Realzadores en la voz del autor y una notable pobreza de marcadores en la voz del lector, lo cual indica que la función persuasiva que conduce a la promoción en este género en español está construida sobre la voz del autor y sobre la autoridad que emana de ella. Se observa un claro enfoque sobre el producto (Realzadores en forma de adjetivos evaluativos positivos). En otro trabajo que igualmente analiza géneros de promoción - guías turísticas-, Suau Jiménez (2012b: 147) concluye que, además, la promoción en forma de voz del autor en español se realiza en la lengua a través de sintagmas nominales (... es el principal plato de la cocina cordobesa / el autobús es la única alternativa...), personificaciones (... Huelva no tiene aeropuerto, por lo que los más cercanos son...), una cierta distancia con el lector a través de oraciones impersonales (... uno se encuentra las playas en el mismo corazón de la ciudad...), abundantes adjetivos calificativos positivos (... fantásticas posibilidades / vida nocturna rutilante / amplísima oferta cultural...) y pocas alusiones al lector. Esta conjunción de rasgos característicos del español conforma el patrón interpersonal que hemos aplicado como metodología en la presente investigación.

\section{Promoción turística en internet: las páginas web de hotel}

Internet se ha convertido en una plataforma única para las operaciones turísticas en las que participa activamente el viajero, habiéndose acuñado así el concepto de turismo 2.0 (Bing y otros, 2007; World Tourism Organization y European Travel Commission, España, 2008). Los géneros electrónicos del turismo en formato 2.0 ofrecen una información que está supliendo los servicios tradicionales de intermediarios como las agencias de viaje, y que se pueden encontrar en portales como TripAdvisor, Trivago, Booking, Expedia o Rumbo, en España. A través de ellos, los usuarios encuentran la información que necesitan para organizar y gestionar sus viajes y estancias, con lo cual la forma en la que se diseñan estos nuevos géneros tiene que utilizar estrategias multimodales que apunten hacia la persuasión.

El turismo 2.0, sus cibergéneros y modos o soportes han creado una comunidad del viajero en la cual internet ya no es sólo un canal, ya que la "migración a la web de algunos géneros textuales ha determinado el desarrollo de prácticas comunicativas específicas caracterizadas por la multimodalidad, el uso de hipertextos o la interactividad" (Calvi y Bonomi, 2008: 185). Por lo tanto, internet es en la actualidad uno de los mejores medios para publicitar hoteles, ciudades, regiones y países, en el cual los propios clientes opinan sobre los productos y servicios que se publicitan y que ellos consumen, proporcionando así una información o retroalimentación muy valiosa para la industria turística.

Los sitios web ofrecen información sin intermediarios, desde las propias empresas turísticas, lo cual crea una imagen competitiva e implica al cliente. La competitividad se alcanza así proporcionando valor comercial a través de 
servicios de calidad, como es la información (Yoke y Ab Hamid, 2011). De nuevo incidimos en la internacionalización, que no se dirige ya solo a un público nacional, sino global. Desarrollar una buena relación con el cliente puede aumentar su satisfacción y su lealtad a un producto o servicio. La gestión de esta relación con el cliente es el concepto más importante en el márketing moderno (Kotler y Amstrong, 2010). Por otra parte, el desarrollo de la web 2.0 exige que los negocios y empresas colaboren con sus clientes a través del uso de esta tecnología (Greenberg, 2010). Los sitios web necesitan, por lo tanto, ser rediseñados y actualizados continuamente para satisfacer las demandas de los clientes y del mercado. Como consecuencia de la internacionalización también se necesita traducir estas web de hotel a otras lenguas, sobre todo a las lenguas mayoritarias. Los mecanismos de persuasión que subyacen textualmente deben ser constantemente reevaluados en términos de cultura, lengua y género para adaptarse -localizarse - lo mejor posible a la lengua meta y a sus códigos o patrones preceptivos.

Las páginas o sitios web de hotel no pertenecen estrictamente a la llamada web 2.0, ya que no permiten una interrelación dialógica directa entre lectores/clientes y hotel en la misma web, como ocurre con otras plataformas digitales que sí pertenecen claramente a la generación 2.0: blogs, redes sociales o wikis. Una página web de hotel que no se rediseña o donde no se actualiza la información se definiría como web 1.0, mientras que una web donde la información se va actualizando con los aportes de los usuarios o lectores, aunque estos vengan de otros soportes electrónicos, sí se considera web 2.0. Estas páginas web de hotel tienen una sola voz explícita en su discurso, la del autor (que representa al hotel o cadena hotelera), quien ha de tener en cuenta e implicar al lector/cliente en sus estrategias discursivas de persuasión.

La voz del lector/cliente y sus preferencias, preguntas o deseos de tipo comercial, es decir, sus necesidades sociocognitivas, llegan a través de otros cibergéneros o modos (Herring, 2011; Thurlow y Mroczek, 2011) como son las reseñas, los comentarios o los cuestionarios, a menudo en un espacio de la propia web del hotel destinado a tal efecto, o a través de foros de viajeros, redes sociales o portales. Solo a través de estos el hotel/autor de la web puede detectar la satisfacción del producto turístico "hotel" y el efecto de las estrategias discursivas desarrolladas en la web sobre los lectores/clientes para reevaluarlas y rectificarlas, una práctica necesaria en el mantenimiento del control de calidad de las webs comerciales (Law, Qi y Buhalis, 2010; Koskensalo, 2012; Suau-Jiménez, 2014a). Se establece así una especie de diálogo bigenérico no explícito, es decir, un diálogo que utiliza más de un género para su comunicación, una característica de las webs de hotel 2.0. Este es el género que se estudia en el presente trabajo, contrastándolo con las fichas de hotel de los portales Booking y TripAdvisor.

\section{El entramado persuasivo en las webs de hotel: funciones retóricas y discurso interpersonal}

El discurso persuasivo de las webs de hotel forma un entramado de funciones retóricas y de discurso interpersonal. Es cierto que las funciones retóricas como la descripción, la explicación, la evaluación y la recomendación actúan al servicio de la función persuasiva. Sin embargo, el discurso interpersonal que se basa en la relación autor-lector, expresado en el propio texto a través de determinados marcadores, es esencial, ya que sirve como correa de transmisión de valores socioculturales y estéticos, y en última instancia, comerciales, implicando al propio lector en el discurso y haciendo que éste se sienta directamente aludido (Dolón, 2012; Suau-Jiménez, 2012b). De esta forma, el autor guía al lector de una página web de hotel y es su interlocutor, utilizando diferentes estrategias según un propósito predeterminado. El discurso interpersonal 
de las webs hoteleras estaría implícito en lo que Perton (2010: 18) denomina "ego-targeting" y describe como

[...] la manera en que un escritor implica al lector en el texto. En la sociedad individualista en que vivimos, casi cada persona quiere servicios y productos para sentirse mejor y tratar a estas personas como si fueran las únicas en el mundo y ofrecerles un tratamiento personal les satisface. Para lograr esto, en la mayoría de los textos turísticos el autor se dirige al lector directamente para que el lector se sienta más especial.

Supone ya un punto de partida compartido por diversos investigadores el hecho de que la función persuasiva, compuesta por estructuras retóricas pero también por discurso interpersonal, sea esencial en los géneros del turismo (MapeIli, 2008; Pierini, 2007, 2009; Calvi, 2010; Edo-Marzá, 2012; Suau-Jiménez, 2012a, 2012b). Si se publicita un hotel obviando o mermando este entramado, el resultado es un texto pobre, basado sobre todo en datos objetivos como situación, número y tipo de habitaciones, servicios y precios, etc., junto con alguna función retórica básica como la descripción, y poco más, eliminando una información más personal y rica, con valores específicos que ofrece el hotel y que el turista 2.0, un individuo formado cultural y socialmente, busca cada vez más, como indica Calvi (2010: 19):

Mientras que en el área de la gestión se emplean formas discursivas parecidas a las de otras actividades comerciales, la comunidad de los expertos del viaje elabora un lenguaje original cuando se dirige al público, para atraerlo, informarlo y dirigir su mirada, ejerciendo un significativo impacto social.

Así pues, no ofrecer una traducción de calidad en la propia web del hotel supone el riesgo de dañar la función persuasiva y, por lo tanto, la eficacia de la propia web, ya que se habrá reducido su utilidad, fundamentada en parámetros de funcionalidad y usabilidad (Law, Qi y Buhalis, 2010: 297). Cuando esto ocurre en una web de hotel, el vacío informativo es sustituido, bien por traductores automáticos deficientes o por portales de servicios de intermediarios tipo Booking o TripAdvisor que solamente ofrecen una ficha escueta con los datos objetivos del hotel, pero no tienen en cuenta la persuasión y el aparato discursivo que le debería acompañar. Cuentan únicamente con un apartado de comentarios de otros viajeros que a través del boca a boca intentan alcanzar el objetivo persuasivo que el hotel busca, y que comentaremos más adelante.

Sabemos que la traducción de calidad de una web está sujeta a factores de tiempo, de presupuesto y/o de problemas técnicos (Bass, 2006: 70-76). Sin embargo, tanto en el diseño de una página web como en su traducción a otras lenguas es donde el hotel, a través de la voz del autor y del discurso interpersonal, puede verter su especificidad y los valores que quiere promocionar. Esto difícilmente se consigue por el método boca a boca de comentarios o reseñas de viajeros, que suelen ser escuetos y utilizar solo pequeñas frases y adjetivos valorativos. Sí pueden servir, en cambio, como fuente de retroalimentación para el necesario rediseño de la web y su adaptación a las expectativas de los clientes.

\section{Análisis del discurso interpersonal traducido en las webs de hoteles}

De los 200 hoteles de gama alta, media y baja analizados en nuestro corpus, sólo 20 ofrecen traducción directa en su página web: 11 de Estados Unidos y 9 de Reino Unido. De estos, todos los hoteles de Reino Unido son de gama alta, mientras que en Estados Unidos 5 pertenecen a la categoría alta y los 6 a la categoría media. A continuación detallamos los datos obtenidos respecto a la traducción de sus webs (ver figuras 1 y 2).

Según este muestreo, en el ámbito de la hostelería se dan distintas estrategias de promoción:

a. Por un lado, un 10\% de los hoteles, generalmente de categoría alta o media, invierte en una página web de calidad, con un diseño que incluye un texto discursivamente rico 


\section{FIGURA 1}

(Suau-Jiménez y Ramírez-Polo, 2014)

\begin{tabular}{c|c|c|c|c|c} 
& EE.UU. & $\begin{array}{c}\text { TRADUCCIÓN } \\
\text { AL ESPAÑOL }\end{array}$ & $\begin{array}{c}\text { TRAD POR GOOGLE } \\
\text { TRANSLATE }\end{array}$ & $\begin{array}{c}\text { SOLO PÁGINA } \\
\text { PRINCIPAL }\end{array}$ & $\begin{array}{c}\text { TRAD A MÁS DE } \\
\text { UN IDIOMA }\end{array}$ \\
\hline Alta & $45,61 \%$ & $8,77 \%$ & & 2 & 2 \\
\hline Media & $31,58 \%$ & $10,53 \%$ & 1 & & \\
\hline Baja & $22,81 \%$ & & & & \\
\hline TOTAL & $\begin{array}{c}\mathbf{1 0 0 , 0 0 \%} \\
(\mathbf{1 0 0} \text { hoteles })\end{array}$ & $\mathbf{1 9 , 3 0 \%}$ & & & \\
\hline
\end{tabular}

\section{FIGURA 2}

(Suau-Jiménez y Ramírez-Polo, 2014)

\begin{tabular}{c|c|c|c|c|c} 
& REINO UNIDO & $\begin{array}{c}\text { TRADUCCIÓN } \\
\text { AL ESPAÑOL }\end{array}$ & $\begin{array}{c}\text { TRAD POR GOOGLE } \\
\text { TRANSLATE }\end{array}$ & $\begin{array}{c}\text { TRAD SOLO PÁGINA } \\
\text { PRINCIPAL }\end{array}$ & $\begin{array}{c}\text { TRAD A MÁS DE } \\
\text { UN IDIOMA }\end{array}$ \\
\hline Alta & $43,33 \%$ & $15,00 \%$ & 2 & & 4 \\
\hline Media & $33,33 \%$ & & & \\
\hline Baja & $23,33 \%$ & & & \\
\hline TOTAL & $\begin{array}{c}\mathbf{1 0 0 , 0 0 \%} \\
(\mathbf{1 0 0} \text { hoteles })\end{array}$ & $\mathbf{1 5 , 0 0 \%}$ & & & \\
\hline
\end{tabular}

en estrategias retóricas y discurso interpersonal, imágenes, $y$, a veces, música o vídeo y traducción a otras lenguas. Estas webs contienen una voz explícita del autor, que representa al hotel mismo, y que se dirige al lector/cliente implicándolo en el discurso.

b. Por otro, hay hoteles, tanto de categoría alta como media o baja, que no invierten en una página web de calidad sino que tan solo contienen una ficha descriptiva con un discurso pobre promocionalmente hablando. Estos hoteles habitualmente se publicitan a través de portales de intermediarios como Booking o TripAdvisor y sustituyen la voz del autor y por tanto la persuasión por el "boca a boca", a través de los comentarios de otros viajeros, en un lugar destacado en la entrada digital del hotel.

A continuación analizamos cualitativamente el discurso interpersonal de varias de estas traducciones del inglés al español en webs de hoteles de categoría alta y media según el marco metodológico y el patrón interpersonal que incluye las voces del autor y del lector (White, 2003; Hyland, 2008) vehiculando así la función persuasiva. Se han incorporado al patrón interpersonal las modificaciones resultantes de varios estudios sobre géneros del turismo 2.0 en español, que han servido para ajustar el tipo de marcadores en ambas voces, autor y lector (Suau-Jiménez, 2012a, 2012b).

1. Hotel Runnymede-on-Thames, Surrey, Reino Unido, 4 estrellas: http://www.runnymedehotel.com/languages/espagnol/

Bienvenido al Runnymede-on-Thames, un maravilloso hotel de 4 estrellas en el sur de Inglaterra, cerca de Londres. Ubicado en Egham, Surrey, a orillas del río Támesis cerca de Windsor. Estamos a pocos minutos del Aeropuerto de Heathrow y cerca de Londres, 
con cómodo acceso a la ciudad por carretera y tren, de modo que contamos con la ubicación ideal para reuniones y eventos, así como para escapadas de fin de semana. Nuestro hotel para toda la familia se encuentra muy cerca de las principales atracciones históricas y parques temáticos del Reino Unido, además de disponer de fantásticas vistas. Pero no necesita salir del hotel para pasarlo en grande, aquí hay mucho por hacer: paseos por la ribera, un galardonado spa, una fantástica cena junto al río y mucho más.

\section{Llámenos a cualquier hora al teléfono..}

Descanse junto al Támesis.

Venga y alójese en el espléndido hotel Runnymede-on-Thames en Surrey. Descansará estupendamente por la noche en nuestras preciosas habitaciones de hotel de lujo y disfrutará todavía más cuando se despierte. Puede pasear, dar de comer a los patos, patronear una barca, mimarse en nuestro spa, jugar al tenis, nadar en una elegante piscina de exterior o visitar el Castillo de Windsor y las sorprendentes atracciones del entorno.
¿Cuál de nuestras fabulosas habitaciones de hotel de lujo es la adecuada para usted? Disponemos de todos los tipos imaginables, incluidas las habitaciones ejecutivas, para familias o para discapacitados. De manera que si está alojado para reunirse con clientes y compañeros de trabajo, pasar tiempo con su familia o con alguien verdaderamente especial, en el runnymede on thames encontrará la habitación que desea.

Duerma como un bebé en nuestras fabulosas camas Hypnos con un mullido edredón de pluma y, si desea uno hipoalergénico, no tiene más que pedirlo. Su habitación dispone de todos los servicios que espera: aire acondicionado con control individual, Wi-Fi, televisión interactiva con películas previa petición y radio, minibar, albornoces y zapatillas, secador de pelo, plancha y tabla de planchar. El servicio de habitaciones está disponible las 24 horas para que pueda beber o comer algo en cualquier momento del día o de la noche, si le entra hambre. El servicio de atención al huésped está a su disposición para cualquier cosa adicional que pudiera necesitar: Ilame en cualquier momento para pedir lo que desee.

\begin{tabular}{|c|c|}
\hline \multicolumn{2}{|c|}{ VOZ DEL AUTOR (explícita en el discurso) } \\
\hline MARCADORES & EJEMPLOS \\
\hline Realzadores descriptivos & $\begin{array}{l}\text { “maravilloso hotel”, "cómodo acceso", "ubicación } \\
\text { ideal”, "fantásticas vistas", "fantástica cena”, } \\
\text { "espléndido hotel”, "preciosas habitaciones", } \\
\text { "elegante piscina", "sorprendentes atracciones", } \\
\text { "fabulosas habitaciones de hotel de lujo", } \\
\text { "fabulosas camas", "mullido edredón” }\end{array}$ \\
\hline Automención del autor & $\begin{array}{l}\text { "estamos a pocos minutos", "nuestro hotel”, } \\
\text { "Ilámenos", "contamos", "disponemos de..", } \\
\text { "nuestras camas", "nuestras camas" }\end{array}$ \\
\hline Actitud del autor & $\begin{array}{l}\text { "muy cerca de", "estupendamente", "alguien } \\
\text { verdaderamente especial”, "mucho por hacer" }\end{array}$ \\
\hline
\end{tabular}




\begin{tabular}{|c|c|}
\hline MARCADORES & EJEMPLOS \\
\hline Compromiso con el lector & $\begin{array}{l}\text { "bienvenido", "descansará”, "no necesita salir", } \\
\text { "disfrutará", "puede pasear....dar de comer.... } \\
\text { patronear...mimarse....nadar...visitar", "si está } \\
\text { alojado", "para que pueda comer o beber”, "si } \\
\text { le entra hambre", "a su disposición”, "cualquier } \\
\text { cosa que pudiera necesitar", "para lo que desee”, } \\
\text { "encontrará", "desea” }\end{array}$ \\
\hline Directivos & $\begin{array}{l}\text { "duerma” "no tiene más que pedirlo", "descanse”, } \\
\text { "venga”, "alójese”, "Ilame”, "llámenos”, "no tiene } \\
\text { más que pedirlo” }\end{array}$ \\
\hline Preguntas & $\begin{array}{l}\text { ¿Cuál de nuestras fabulosas habitaciones de hotel } \\
\text { de lujo es la adecuada para usted? }\end{array}$ \\
\hline
\end{tabular}

2. Hotel Master Robert, Londres, Reino Unido, 3 estrellas: http://www.masterrobert.co.uk/sp/

Nuestra más cálida bienvenida al hotel Master Robert, su hogar ideal cuando esté lejos de casa.

El hotel Master Robert es desde hace mucho tiempo la opción favorita para clientes de negocios, personas que viajan hacia y desde el aeropuerto de Heathrow y aquellas que visitan Londres. El hotel es renombrado por la amabilidad de su personal y su tradicional confort inglés. Un pub inglés tradicional en la zona, un restaurante galardonado con el premio RAC y las instalaciones para banquetes y conferencias aportan aún más atractivo al hotel, un establecimiento de importancia histórica.

El hotel es uno de los más cercanos a Heathrow y dispone de amplias habitaciones y salas de conferencias. Tiene capacidad para acoger a 130 personas en sus salas de conferencias. También cuenta con espacio para la celebración de bodas y ofrece un menú adaptado a la cocina local o bien a la asiática. Los visitantes tienen fácil acceso al Aeropuerto de Heathrow, ya que el hotel se encuentra muy próximo. Los lugares importantes cercanos al hotel son Feltham, el parque Stockley, Southall, Legoland, Isleworth, Uxbridge, Hounslow, Twickenham, los jardines de Kew, el Castillo de Windsor, Harrow y Hounslow. Numerosas compañías internacionales están ubicadas en Uxbridge y el Civic Centre, que no está asimismo lejano. Se trata también de un magnífico lugar para viajeros de negocios. Diseñado para el placer pero equipado para los negocios, el Master Robert Hotel es desde hace mucho tiempo la opción favorita para los clientes corporativos, las personas que viajan hacia o desde el aeropuerto de Heathrow, y, del mismo modo, aquellas que visitan Londres y los destinos turísticos y de ocio de los alrededores.

Famoso en el sector hotelero de Heathrow por sus amplias habitaciones, excelente servicio y espacioso aparcamiento, el hotel está magníficamente equipado para satisfacer sus necesidades (haga clic aquí para ver las instalaciones de las habitaciones). El aparcamiento tiene un coste de $£ 5,00$ por coche/día sujeto a disponibilidad. Heathrow es conocido por ser uno de los aeropuertos más concurridos del Reino Unido y del mundo. Aparcar resulta a veces complicado, pero ahora usted puede disfrutar de la tranquilidad de nuestro Paquete "Aparcar y Volar". 
Le ofrecemos servicio de aparcamiento en nuestro hotel, donde podrá obtener aparcamiento gratuito durante 15 días cuando se aloje al menos una noche (sujeto a Tér- minos y Condiciones). Podrá obtener más información sobre esta oferta contactando con el hotel, bien por teléfono o por correo electrónico.

\begin{tabular}{|c|c|}
\hline \multicolumn{2}{|c|}{ VOZ DEL AUTOR (explícita en el discurso) } \\
\hline MARCADORES & EJEMPLOS \\
\hline Realzadores descriptivos & $\begin{array}{l}\text { "más cálida bienvenida”, "hogar ideal”, "opción } \\
\text { favorita", "famoso", "amplias habitaciones, } \\
\text { excelentes servicios y espacioso aparcamiento", } \\
\text { "renombrado", "tradicional confort”, "menú } \\
\text { adaptado a la cocina local o a la asiática”, } \\
\text { "restaurante galardonado", "más atractivo", } \\
\text { "importancia histórica", "amplias habitaciones", } \\
\text { "fácil acceso", "lugares importantes cercanos", } \\
\text { "magnífico lugar para viajeros de negocios", } \\
\text { "diseñado para el placer pero equipado para los } \\
\text { negocios" }\end{array}$ \\
\hline Automención del autor & "nuestra más cálida bienvenida" \\
\hline Actitud del autor & "magníficamente equipado" \\
\hline
\end{tabular}

\section{VOZ DEL LECTOR (implícita en el discurso)}

\begin{tabular}{|l|l}
\hline MARCADORES & EJEMPLOS
\end{tabular}

Compromiso con el lector "su hogar ideal”, "cuando esté lejos de casa”, "usted puede disfrutar de la tranquilidad", "cuando se aloje", "podrá obtener más información"

\section{Análisis discursivo interpersonal de fichas de hoteles de EEUU y Reino Uni- do en portales de servicios turísticos en español}

Tras un rastreo en internet, hemos comprobado que los portales y metabuscadores de servicios turísticos que ofrecen una ficha con información textual en español sobre hoteles son Booking, TripAdvisor, Trivago, Expedia y Rumbo, en España.

Nuestro análisis se limita a los portales Booking y TripAdvisor, porque ofrecen más información que el resto de portales observados. Analizamos a continuación, a través de la misma metodología del discurso interpersonal, sus fichas descriptivas en dos ejemplos de cada uno de estos dos portales para el mismo hotel:
1. Hotel Park Plaza Westminster Bridge London, 4 estrellas

Fuente: www.booking.com

El Park Plaza Westminster Bridge London se encuentra frente al Parlamento y el Big Ben, en la ribera sur de Londres. Además, está cerca de la noria London Eye, el acuario, así como diversos restaurantes y teatros.

Las habitaciones son amplias, modernas y lujosas. Cuentan con aire acondicionado, TV de pantalla plana, escritorio amplio, minibar, nevera y caja fuerte. También hay disponibles estudios y apartamentos.

El Park Plaza Westminster Bridge está situado junto al río Támesis, a 500 metros del London Eye y de la estación London Waterloo. 
La estación de metro de Westminster se encuentra a pocos pasos

El Westminster Bridge Park Plaza alberga la moderna y galardonada Brasserie Joel, el Ichi Sushi \& Sashami y el IWB Lounge \& Patisserie, que sirve el tradicional té de la tarde. Por su parte, el Primo Bar ofrece entrete-
nimiento cada noche
El establecimiento cuenta con un gimnasio abierto las $\mathbf{2 4}$ horas totalmente equipado, una piscina de 15 metros con sauna y sala de vapor, y el recién inaugurado spa Mandara, que ofrece una amplia gama de tratamien- tos de lujo

\begin{tabular}{l|l}
\hline \multicolumn{2}{l}{ VOZ DEL AUTOR (explícita en el discurso) } \\
\hline MARCADORES & EJEMPLOS \\
\hline Realzadores & "las habitaciones son amplias, modernas y lujosas", \\
& "la moderna y galardonada Brasserie Joel", "amplia \\
& gama de tratamientos de lujo" \\
\hline
\end{tabular}

VOZ DEL LECTOR (implicita en el discurso): inexistente

Fuente: www.tripadvisor.com

Disfrute de la situación de nuestro moderno hotel cercano a Westminster y el London El recientemente inaugurado hotel Park Plaza Westminster Bridge London se encuentra en el South Bank de Londres, a unos minutos a pie de los principales iconos de la ciudad, incluyendo el Big Ben y las Casas de Parlamento, el London Eye y el London Aquarium. La situación de nuestro hotel cerca del London Eye, el London Aquarium y el National Theatre hace del Park Plaza el alojamiento perfecto para huéspedes en viaje tanto de negocios como de ocio. Nuestro moderno hotel de diseño de Westminster Bridge cuenta con amplias habitaciones, incluidas 65 suites y áticos, y 500 estudios. Los restaurantes del hotel de Westminster inclu- yen cinco distintivas experiencias de gastronomía y entretenimiento, incluido el aclamado restaurante francés Brasserie Joël.

Entre los servicios de nuestro hotel de Londres Park Plaza Westminster Bridge se encuentran un salón de 1.200 metros sin columnas, 31 salas de reuniones adicionales, un salón ejecutivo y Wi-Fi gratuito en todo el hotel. También se encuentra en el hotel el primer y único Spa y sala de fitness Mandara de Europa con piscina de 15 metros. El spa ofrece los mejores tratamientos y productos del spa Mandara, Elemis Face and Body Therapies y Bliss Nails \& Waxing. Las estaciones de metro Waterloo y Westminster se encuentran a 5 minutos a pie de este hotel de Westminster Bridge.

\begin{tabular}{|c|c|}
\hline \multicolumn{2}{|c|}{ VOZ DEL AUTOR (explícita en el discurso) } \\
\hline MARCADORES & EJEMPLOS \\
\hline Realzadores descriptivos & $\begin{array}{l}\text { “moderno hotel cercano a”, "alojamiento perfecto”, } \\
\text { “moderno hotel de diseño”, "amplias habitaciones”, } \\
\text { "aclamado restaurante y brasserie Joel”, "los } \\
\text { mejores tratamientos y productos” }\end{array}$ \\
\hline Automención del autor & "nuestro hotel”, “nuestro moderno hotel” \\
\hline
\end{tabular}




\begin{tabular}{l|l}
\hline \multicolumn{2}{|l}{ VOZ DEL LECTOR (implícita en el discurso) } \\
\hline MARCADORES & EJEMPLOS \\
\hline Directivos & "disfrute de..." \\
\hline
\end{tabular}

2. St. Giles London, 3 estrellas Fuente: www.booking.com

El St Giles está situado en el West End de Londres, a menos de 5 minutos a pie de la estación de metro Tottenham Court y de las tiendas de Oxford Street. El teatro Dominion se encuentra a solo 3 minutos a pie, y el hotel ofrece 3 opciones de restauración: el Sage \& Chili (fusión de comida asiática e italiana), el Hudson's House Café Lounge Bar, y el VQ, una animada cafetería y restaurante abierta las 24 horas.

Tanto Covent Garden como el Museo Británico están a unos 10 minutos a pie del St Giles Hotel \& Leisure Club. La Galería Nacional se halla a menos de 15 minutos a pie.

Debajo del St Giles está el centro de fitness y bienestar más grande de Londres. Los huéspedes recibirán una entrada con descuento para las instalaciones de ocio de YMCA, que incluyen una piscina y un gimnasio.

El bar suele estar abierto hasta tarde todas las noches.

Todas las habitaciones del St Giles son luminosas y están equipadas con baño privado con ducha, TV y un set gratuito de té y café. Además, tienen una decoración clásica y cuentan con caja fuerte y secador de pelo. Se ofrece conexión a internet.

\begin{tabular}{l|l}
\hline \multicolumn{2}{l}{ VOZ DEL AUTOR (explícita en el discurso) } \\
\hline MARCADORES & EJEMPLOS \\
\hline Realzadores & "animada cafetería y restaurante", "el centro de \\
& $\begin{array}{l}\text { fitness más grande de Londres", "habitaciones } \\
\text { luminosas" }\end{array}$ \\
\hline
\end{tabular}

VOZ DEL LECTOR (implícita en el discurso): inexistente Fuente: www.tripadvisor.com

Uno de los hoteles más céntricos del West End de Londres, St Giles Hotel se encuentra en el cruce de Tottenham Court Road y Oxford
Street. A tan solo un paseo a pie de las mejores calles comerciales de Londres así como a otras atracciones turísticas y teatros.

\begin{tabular}{l|l}
\hline \multicolumn{2}{l}{ VOZ DEL AUTOR (explícita en el discurso) } \\
\hline MARCADORES & EJEMPLOS \\
\hline Realzadores & "uno de los hoteles más céntricos", "a tan solo un \\
& paseo a pie de las mejores calles comerciales" \\
\hline
\end{tabular}

VOZ DEL LECTOR: inexistente 


\section{Resultados}

Los resultados del análisis interpersonal de las páginas web de hotel traducidas muestran un discurso más rico, al contrario que el de las fichas de portales o metabuscadores en español. Se observa que los marcadores más utilizados del patrón interpersonal a través de las voces del autor y del lector (Hyland, 2008) coinciden con el discurso interpersonal identificado para los géneros del turismo en español (Mapelli, 2008; Pierini, 2009; Suau-Jiménez, 2012a, 2012b) como los realzadores y los marcadores de compromiso con el lector, respectivamente, aunque también la automención y los marcadores de actitud, así como los directivos y las preguntas. A través de los realzadores se transmiten los valores que caracterizan al hotel y que conforman su especificidad y, por lo tanto, parte de su estrategia de márketing, como la estética, la comodidad de su situación, la calidad de su gastronomía o de sus atracciones, la elegancia de su piscina y la comodidad de sus camas en el Runnymede-onThames o en el Master Robert la sensación de hogar, la amplitud de sus habitaciones, el confort típico británico, su cocina europea y asiática, su renombre histórico y su disponibilidad para viajeros por placer o por negocios.

Por otra parte, los marcadores de compromiso con el lector implican al cliente en el propio discurso, convirtiéndolo en interlocutor de la voz del establecimiento y en destinatario de lo que el hotel ofrece. En el Runnymede-on-Thames esto se consigue mediante alusiones a la bienvenida al descanso, al disfrute, a las múltiples opciones que el hotel ofrece (comer, patronear, mimarse, nadar, visitar), y especialmente al servicio personalizado (para que pueda comer o beber, si le entra hambre, a su disposición, cualquier cosa que pudiera necesitar, para lo que desee). El hotel Master Robert, aunque en menor medida, hace también uso de estos marcadores, siendo su objetivo específico la sensación de hogar y la tranquilidad.
Sin embargo, según el resultado del análisis de dos hoteles a través de dos portales, Booking y TripAdvisor, observamos la práctica desaparición del discurso interpersonal, especialmente en los marcadores de compromiso con el lector, cuya voz es inexistente. Tan solo se utilizan algunas estructuras o funciones descriptivas respecto a la situación del hotel. Aparecen algunos realzadores en forma de adjetivos evaluativos, que es el único marcador que se incluye en las fichas, pero se han reducido al mínimo, posiblemente para cumplir con las dimensiones del formato impuesto por la ficha, y se utilizan tan solo para realzar el producto/servicio, pero sin transmitir características específicas como ocurre con las webs traducidas. Son adjetivos evaluativos que forman sintagmas nominales con muy reducidos contenidos específicos ("habitaciones luminosas", "moderno hotel cercano a”, "alojamiento perfecto", "los mejores tratamientos y productos"). Se han eliminado todas las alusiones directas al lector, con lo cual no se implica al cliente en el propio discurso del hotel ni se le hace interlocutor de su comunicación.

En todos los casos analizados a través de los portales Booking y TripAdvisor aparece una sección destinada a comentarios de los viajeros, dirigidos a los clientes a través de la estrategia del boca a boca para sustituir el diálogo implícito que no aparece en la ficha. En TripAdvisor, el apartado de los comentarios está mejor diseñado que en Booking: el hotel St Giles muestra 1917 opiniones de viajeros en formato de cita, con toda clase de información, positiva y negativa y un cuadro de valoración del hotel con posibilidades de excelente, muy bueno, bueno, regular y malo. En el Hotel Master Robert de Londres, también en TripAdvisor, aparecen 237 opiniones también con información positiva y negativa y sin estar clasificada por conceptos como servicio, ubicación, etc.

El portal Booking, con menor información valorativa y de comentarios, incluye una pun- 
tuación concreta para cada hotel, basada en un cálculo de las puntuaciones de los viajeros, y solo un extracto de comentarios sobre aspectos como situación, desayuno, tamaño de las habitaciones o servicio. Los otros dos portales examinados, Expedia y Rumbo, ofrecen fichas similares a Booking, con funciones descriptivas básicas y apoyo a través de comentarios de viajeros, a veces con un enlace a TripAdvisor.

\section{Conclusiones y futuras investigaciones}

Una de las ventajas que se observa en la traducción de calidad de una web de hotel es el cuidado de la imagen del establecimiento a través del contenido textual. Se consigue mediante la preservación del discurso interpersonal, con un uso adecuado de este patrón según el género, la lengua y la disciplina. El fuerte contenido apelativo hacia el lector/cliente marca la mayor diferencia con las fichas de portales/buscadores, incluso cuando estos incluyen comentarios de viajeros para suplir dicha apelación. Una traducción de calidad o traducción pragmática en la propia web del hotel debería ir, pues, paralela a la imagen que el hotel quiere transmitir y que se construye de forma multimodal y multisemiótica, pero donde el texto es un elemento clave de la persuasión, que llega al lector/cliente y establece un diálogo implícito a través de un discurso interpersonal cuidadosamente elaborado y traducido, teniendo en cuenta el patrón de la lengua origen y el patrón de la lengua meta (Suau-Jiménez, 2011). La imagen de marca del hotel se construye tanto con elementos visuales y auditivos (imágenes, vídeos, música ambiental) como con un texto que incluya y transmita las características específicas que un hotel está interesado en promocionar y que crean una diferencia competitiva con otros establecimientos. En las fichas de portales, sin embargo, esta información, que sigue un mismo patrón estructural para todos los hoteles, hay que buscarla en los comentarios de otros viajeros, que no siempre están recogidos de forma completa, sino que aparecen a menudo como un extracto, tal como ocurre con el portal Booking. Como sugieren varios autores (Pym, 2004; Bass, 2006; Pierini, 2007), la traducción debe ser parte ineludible de la localización de una web, especialmente si ésta actúa como imagen de un establecimiento concreto. A esto se añade la propuesta de Dunne (2006) de unir los conceptos globalización, internacionalización, localización y traducción, como garantía de calidad en la promoción de productos y servicios.

Por lo tanto, la conclusión iría en el sentido de que sí es necesaria la traducción de las webs hoteleras si se quiere ofrecer un mayor soporte de la función persuasiva a través de la especificidad de cada hotel, así como de la implicación del lector en el diálogo que se establece. Se trataría de que la industria hotelera fuera consciente de esta necesidad, que puede conducir a un mejor resultado de sus ventas. Igualmente, quizá son necesarias acciones promovidas por asociaciones profesionales de traductores que impliquen a sectores industriales como el turismo para, de forma colectiva, establecer protocolos que den como resultado un mejor diseño y traducción de las webs hoteleras, lo cual puede redundar en un aumento de su actividad económica.

El presente trabajo abre nuevas vías de investigación desde el marco teórico y metodológico del discurso interpersonal o interpersonalidad aplicado a la traducción de géneros de promoción de productos y servicios. Este paradigma metodológico, procedente de los géneros académicos, supone una perspectiva novedosa con una interesante aplicabilidad a ámbitos profesionales y comerciales donde la persuasión del cliente como forma de promoción es esencial. Un ejemplo serían los sitios web de pequeñas empresas con vocación internacional, que necesitan ofrecer una traducción de calidad para que no se traicione su imagen de marca (Medina Reguera y Ramírez Delgado, 2014). Igualmente, este trabajo contiene una implicación teórica y traductológica, ya que amplía el espectro meto- 
dológico de la localización de páginas web como concepto clave en la traslación de una cultura a otra (Jiménez Crespo, 2008, 2013), al incluir el discurso interpersonal como elemento necesario de dicha localización.

\section{Bibliografía citada}

Bass, Scott, 2006: "Quality in the Real World" en Keiran J. Dunne (ed.): Perspectives on Localization, Amsterdam: John Benjamins, 69-84.

Bing, Pan, Tanya Maclaurin y John C. CRotTs, 2007: "Travel blogs and the implications for destination marketing", Journal of Travel Research 46, 1, 35-45.

Bonomi, Milin, 2011: "El lenguaje del turismo en la web 2.0" en Elena Liverani y Jordi Canals (eds.): El discurso del turismo. Aspectos Lingüísticos y Variedades Textuales, Trento: Tangram Edizioni Scientifiche.

Calvi, Maria Vittoria y Milin Bonomi, 2008: "El lenguaje del turismo: de los textos especializados a la Comunidad del Viajero" en Carmen Navarro, Rosa Rodríguez Abella, Francesca Dalle Pezze y Renzo Mıotтı (eds): La comunicación especializada. Linguistic Insights Vol. 87, Bern: Peter Lang.

Calvi, Maria Vittoria, 2010: "Los géneros discursivos en la lengua del turismo: una propuesta de clasificación”, Ibérica 19, 9-32.

Chen, Injazz J. y Karen Popovich, 2003: "Understanding customer relationship management (CRM) - People, process and technology", Business Process Management Journal, vol. 9(5), 672-688.

Cheng, Aw Yoke y Noor R. Aв Hamid, 2011: "The Usability of Hotel Websites in Managing Tourist Expectations: A Customer Relationship Perspective", IPEDR vol.10, IACSIT Press, Singapore, 43-47.

I Congreso Internacional de Traducción, Financiera, Comercial e Institucional, 2014. Grupo COMENEGO. Universidad de Alicante, 28-31 mayo 2014 [http://dti.ua.es/es/comenego/congreso-traduccion-economica/congreso-internacional-de-traduccion-economica-comercial-financiera-e-institucional.html, fecha de consulta: 6 junio 2014].

Crismore, Avon, Raija Markanen y Margaret SteffenSEN, 1993: "Metadiscourse in persuasive writing", Written Communication 10 (1), 39-71.

Dolón, Rosana, 2012: "El niño como actor social en el discurso turístico: un estudio de análisis crítico de la construcción discursiva de una identidad de consumidor" en Julia Sanmartín Sáez (ed.): Discurso Turístico e Internet, Madrid: Iberoamericana/Vervuert, 155-186.

Dunne, Keiran J., 2006: "Introduction: A Copernican Revolution" en Keiran J. Dunne (ed.): Perspectives on Localization, Amsterdam: John Benjamins, 1-11.

Durán Muñoz, Isabel, 2011: El trabajo ontoterminográfico aplicado a la traducción de textos del turismo de aventura (español, inglés, alemán): fases de preparación, elaboración y edición, Málaga: Servicio de Publicaciones de la Universidad de Málaga.

Durán Muñoz, Isabel, 2012: "Caracterización de la traducción turística: problemas, dificultades y posibles soluciones", Revista de Lingüística y Lenguas Aplicadas, vol. 7, 103-113.

Edo MarzA, Nuria, 2012: "Páginas web privadas e institucionales: el uso de la adjetivación en un corpus inglés-español de promoción de destinos turísticos" en Julia Sanmartín Sáez (ed.:) Discurso turístico e Internet, Madrid: Iberoamericana/Vervuert, 51-80.

Esselink, Bert, 2000: A Practical Guide to Localization, Amsterdam: John Benjamins.

Greenberg, Paul, 2010: "The impact of CRM 2.0 on customer insight", Journal of Business \& Industrial Marketing, vol. 25, 6, 410-419.

Halliday, Michael, 1985: An Introduction to Functional Grammar, London: Edward Arnold. 
HerRing, Susan, 2011: "Commentary", en Crispin Thurlow y Christine Mroczek (eds.): Digital discourse: Language in the new media, New York: Oxford University Press, 340-347.

Hyland, Kenneth y Polly Tse, 2004 "Metadiscourse in Academic Writing: A Reappraisal", Applied Linguistics 25/2, 156-177

Hyland, Kenneth, 2005: Metadiscourse, London: Continuum.

Hyland, Kenneth, 2008: "Disciplinary voices. Interactions in research writing”, English Text Construction 1:1, 5-22.

Jiménez Crespo, Miguel Ángel, 2008: "El proceso de localización web: estudio contrastivo de un corpus comparable del género sitio web corporativo". Tesis Doctoral, Universidad de Granada (España).

Jiménez Crespo, Miguel Ángel, 2011: "To adapt or not to adapt in web localization: A contrastive genre-based study of original and localized legal sections in corporate websites", The Journal of Specialized Translation 15, 2-27.

Jiménez Crespo, Miguel Ángel, 2013: "Translation and web localization”, Abingdon: Routledge.

Koskensalo, Annikki, 2012: "Towards a better professional understanding of the hybrid genre 'tourism websites'”, Proceedings of the 3rd Conference of the Asia Pacific Language for Special Purposes and Professional Communication Association, University of Southern California, Los Angeles, CA.

Kotler, Philip y Gary Amstrong, 2010: Principles of marketing, London: Pearson.

Law, Robert, Shanshan Qı y Dimitrios BuHALIS, 2010: "Progress in tourism management: A review of website evaluation in tourism research", Tourism Management 31, 297-313.

Liverani, Elena y Jordi Canals (eds.), 2011: El discurso del turismo. Aspectos Lingüísticos y Variedades Textuales, Trento: Tangram Edizioni Scientifiche.
Mapell, Giovanna, 2008: "Las marcas de metadiscurso interpersonal de la sección 'turismo' de los sitios web de los ayuntamientos" en $M^{\text {a }}$ Vittoria Calvi, Giovanna Mapelli y Javier Santos (eds): Lingue, culture, economia: comunicazione e pratiche discorsive, Milano, Franco Angeli, 173-190.

Medina Reguera, Ana y Cristina Ramirez Delgado, 2014: "La traducción en la empresa: internacionalización y localización de sitios webs corporativos de empresas andaluzas", comunicación presentada en el I Congreso Internacional de Traducción Económica, Financiera, Comercial e Institucional

[http://dti.ua.es/es/comenego/congreso-traduccion-economica/congreso-internacional-de-traduccion-economica-comercial-financiera-e-institucional.html, fecha de consulta: 3 junio 2014].

Navarro, Carmen, Ma Rosa Rodríguez Abella, Francesca Dalle Pezze y Renzo Mıotti (eds.), 2008: La comunicación especializada en Linguistic Insights, vol. 87, Bern: Peter Lang.

NoBs, Marie Louise, 2003: Expectativas y evaluación en traducción de folletos turísticos: estudio empírico con usuarios reales. Tesis doctoral, Universidad de Granada.

Nord, Christiane, 1997: Translating as a Purposeful Activity: Functionalist Approaches Explained, Manchester: St Jerome.

Perton, Narda, 2010: La traducción de textos turísticos. Tesina, Universiteit Utrecht Vertalen.

PIerInI, Patricia, 2007: "Quality in Web Translation: An Investigation into UK and Italian Tourism Web Sites", The Journal of Specialized Translation, Issue 8

[http://www.jostrans.org/issueo8/art_pierini. php, fecha de consulta: 15 mayo 2014].

PIerinI, Patricia, 2009: "Adjectives in Tourism English on the Web. A corpus-based study", CIRCULO de Lingüística Aplicada a la Comunicación (clac) 40/2009, 93-116, Madrid: Universidad Complutense de Madrid. 
Pym, Anthony, 2004: The Moving Text: Localization, Translation and Distribution, Amsterdam: John Benjamins.

SuAu-Jıménez, Francisca, 2011: "La persuasión a través del metadiscurso interpersonal en el género Página Web Institucional de Promoción Turística en inglés y español" en Maria Vittoria Calvı y Giovanna Mapeluı (eds.): La Lengua del Turismo: Géneros discursivos y terminología, Bern: Peter Lang, Colección: Linguistic Insights, vol. 138, 177-200.

SuAu-Jiménez, Francisca, 2012a: "Páginas web institucionales de promoción turística: el uso metadiscursivo interpersonal en inglés y español" en Julia Sanmartín Saez (ed.): Discurso Turístico e Internet, Madrid: Iberoamericana/Vervuert, 125-154.

SuAu-Jiménez, Francisca, 2012b: "El turista 2.0 como receptor de la promoción turística: estrategias lingüísticas e importancia de su estudio", PASOS, Revista de Turismo y Patrimonio Cultural vol. 6, $n^{\circ}$ 4, número especial, mayo 2012

[http://www.pasosonline.org/Publicados/10412 special/PASOS30_Special_Issue.pdf, fechadeconsulta: 4 noviembre 2013].

SuAu-Jıménez, Francisca, 2014a: "Estrategias discursivas interpersonales en el turismo 2.0: diferencias e implicaciones", comunicación presentada en el Simposio Internacional Discurso y Géneros del Turismo 2.0. Universitat de València, 2-4 abril 2014 [http://www.uv.es/cometval/wikibase/cas/ Simposio/home.html, fecha de consulta: 1 mayo 2014].

SuAu-Jiménez, Francisca, 2014b: "El proceso pre-traductológico en textos de economía a través del análisis metadiscursivo interpersonal: estudio piloto del corpus COMENEGO", Hermeneus. Revista de Traducción e Interpretación, Monográfico Vertere $n^{\circ} 16$, editado por Daniel Gallego.

SuAu-Jiménez, Francisca y Rosana Dolón-Herrero, 2007: "The Importance of Metadiscourse in the genre 'Promotion of Touristic Services and Products': Differences in English and Spanish" en Dita Galová (ed.): Languages for Specific Purposes: Searching for Common Solutions, Cambridge Scholars Publishings, 70-79.
Suau-Jiménez, Francisca y Laura Ramírez-Polo, 2014: "La traducción estratégica de páginas web de hoteles: un elemento necesario para la persuasión del cliente", comunicación presentada en el I Congreso de Traducción Económica, Financiera, Comercial e Institucional

[http://dti.ua.es/es/comenego/congreso-traduccion-economica/congreso-internacional-de-traduccion-economica-comercial-financiera-e-institucional.html, fecha de consulta: 3 junio 2014].

Thurlow, Crispin, 2001: "Language and the Internet” en Mesthrie Rejend y Raymond Asher (eds.): The concise encyclopedia of sociolinguistics, London: Pergamon.

Thurlow, Crispin y Kristine Mroczek (eds.), 2011: Digital discourse: Language in the new media, Oxford: Oxford University Press.

TIA, 2005: "Executive Summaries-Travellers'Use of the Internet, 2004 Edition" [http://www.tia. org/researchpubs/executive_summaries_travellers_use.html. fecha de consulta: 15 noviembre 2013].

White, Peter, 2003: "Beyond modality and hedging: a dialogic view of the language of intersubjective stance", Text 23(2), 2594-2598.

World Tourism Organization (UNWTO) y European Travel Commission (ETC), 2008: Handbook of E-Marketing for Tourism Destination, Spain: WTO and ETC.

Vande Kopple, William J., 1985: "Some exploratory discourse on metadiscourse", College Composition and Communication 36, 82-93. 